\title{
Regulation, policy and user behaviour analysis for telecommunications networks and services
}

\author{
Dimitris Varoutas
}

Accepted: 13 December 2010 / Published online: 13 January 2011

(C) Springer Science+Business Media, LLC 2011

Telecommunications engineering and its economic and business aspects are the research focus of many researchers worldwide. As the mobile market has reached saturation especially in Europe and fiber-to-the-home developments are still late, the analysis of telecommunications networks and services necessitates the mix of regulation, policy and user analysis from a technoeconomic point of view. This special issue aims to support the research in these directions by accommodating original contributions from researchers working in technoeconomics and participating in the Conferences on Telecommunication Techno-Economics (www.ctte-conference.org). The contributions of this special issue span user behaviour analysis to regulation and have been written from engineers and economists, underlying the interdisciplinary and multifaceted character of the telecommunication technoeconomics.

In the article "An econometric analysis of mobile services growth across regions of India" Sridhar provides insights of the mobile services growth in India. Taking into account that India is the third largest mobile market in terms of subscribers, an analysis of factors affecting the mobile services growth is interesting and timely. The paper models the growth of mobile services using technology diffusion analysis and indicates the competition and network effects which drive the mobile services development in India. It is worth noting that the paper challenges the traditional views about mobile growth as it concludes that the traditional factors such as income, population and fixed

\footnotetext{
D. Varoutas $(\bowtie)$

Faculty of Informatics and Telecommunications, School of Sciences, University of Athens, Panepistimiopolis, Ilisia, Athens, Greece 157-84

e-mail: D.Varoutas@di.uoa.gr
} 
line penetration do not have any significant impact on the growth of mobile subscription.

The article "Estimating diffusion and price elasticity with application to telecommunications" by Michalakelis et al. introduces an interesting approach on price elasticity in demand forecasting, by developing a "diffusion-price" model. The authors estimate simultaneously DSL diffusion and prices with data from European countries and provide a way to develop business strategies in terms of product demand and pricing.

Kivi in his article "Diffusion of mobile data in Finland" discusses the interesting issue of mobile Internet or mobile data services adoption by the masses. By using data in Finland during 2005-2006 over an 80-90\% of Finnish mobile subscribers and terminals, he shows a multiplication of total data traffic volumes. However, based on this analysis, the article concludes that the critical mass of users necessary for mass market adoption has not yet been achieved in Finland.

In the article "Hedonic study on mobile telephony market in France: pricing-quality strategies", Karamti and Grzybowski adopt the hedonic price theory in order to analyse the relation between quality and price in the mobile telephony market during 1996-2002 in France. As the quality-adjusted price index declines at about $14 \%$ from 1997 to 1999 while on the remaining period the price decrease tends to flatten, the article claims that the three mobile providers seem to adopt similar pricing behaviors. On the other hand, for the period 2000-2002 neither the quality nor the price indexes show significant changes for the three operators in France. These results are of some consequence, especially after the French antitrust authority decision in December 2005 about the existence of collusion on the mobile market during the same period (2000-2002).

Di Pillo et al. in their article "Asymmetry in mobile access charges: is it an effective regulatory measure?" analyse the competition between interconnected mobile phone networks, under the assumptions of discriminatory retail pricing and non reciprocal access charges. They conclude that if the retail prices are different between calls that terminate on the same network (on net) and calls that terminate on the rival network (off net), the competition is more complex, involving positive network externalities for the incumbent operator.

In the article "Asymmetric competition and collection rates differentials: determinants of prices in international telephone service markets", by Nucciarelli et al., pricing in international telephone service markets is investigated. The authors develop an econometric model to study the impact of different telecommunications-specific and macroeconomic variables over collection rate differentials and traffic imbalance between countries. Data from 24 worldwide national markets strengthen empirical findings from the theoretical analysis and make the econometric model an interesting and flexible instrument for new and efficient-turned telecommunications policies. 
I would like to thank the authors of the articles in this special issue for their contributions that cover several aspects of the broad telecommunications technoeconomic area. Readers and authors' feedback on any aspect of this special issue as well as ideas on how we can further develop and publicize the technoeconomic aspects of telecommunications and networking in order to make it appealing to you, the readers are most welcome at D.Varoutas@di.uoa.gr. 